\title{
H I absorption in 3C 49 and 3C 268.3
}

\section{Probing the environment of compact steep spectrum and GHz peaked spectrum sources}

\author{
A. Labiano ${ }^{1,2}$, R. C. Vermeulen ${ }^{3}$, P. D. Barthel ${ }^{1}$, C. P. O’Dea ${ }^{4}$, J. F. Gallimore ${ }^{5}$, S. Baum ${ }^{6}$, and W. de Vries ${ }^{7}$ \\ 1 Kapteyn Astronomical Institute, Groningen, 9700 AV, The Netherlands \\ e-mail: labiano@astro.rug.nl \\ 2 Space Telescope Science Institute, Baltimore, MD 21218, USA \\ 3 Netherlands Foundation for Research in Astronomy, Dwingeloo, The Netherlands \\ ${ }^{4}$ Department of Physics, Rochester Institute of Technology, Rochester, NY 14623, USA \\ 5 Department of Physics, Bucknell University, Lewisburg, PA 17837, USA \\ ${ }^{6}$ Center for Imaging Science, Rochester Institute of Technology, Rochester, NY 14623, USA \\ 7 Lawrence Livermore National Lab., Livermore, CA 94550, USA
}

Received 19 July 2005 / Accepted 9 October 2005

\section{ABSTRACT}

Aims. Localize and study the redshifted $21 \mathrm{~cm} \mathrm{H} \mathrm{I} \mathrm{absorption} \mathrm{known} \mathrm{to} \mathrm{occur} \mathrm{in} \mathrm{the} \mathrm{subgalactic} \mathrm{sized} \mathrm{compact} \mathrm{steep} \mathrm{spectrum} \mathrm{galaxies} \mathrm{3C} 49$ and $3 \mathrm{C} 268.3$.

Methods. We carried out European VLBI Network UHF band spectral line observations.

Results. We have detected H I absorption towards the western radio lobe of 3C 49 and the northern lobe of 3C 268.3. However, we cannot rule out the presence of similar amounts of H I towards the opposite and much fainter lobes. The radio lobes with detected H I absorption (1) are brighter and closer to the core than the opposite lobes; (2) are depolarized; and (3) are associated with optical emission line gas. The association between the $\mathrm{H} \mathrm{I}$ absorption and the emission line gas, supports the hypothesis that the $\mathrm{H} \mathrm{I}$ absorption is produced in the atomic cores of the emission line clouds. Our results are consistent with a picture in which compact steep spectrum sources interact with clouds of dense gas as they propagate through their host galaxy. We suggest that the asymmetries in the radio and optical emission can be due to interaction of a two sided radio source with an asymmetric distribution of dense clouds in their environment.

Key words. galaxies: active - galaxies: individual: 3C 49 - galaxies: individual: 3C 268.3 - galaxies: quasars: absorption lines radio lines: galaxies - galaxies: jets

\section{Introduction}

The GHz Peaked Spectrum (GPS) and Compact Steep Spectrum (CSS) radio sources make up significant fractions of the extragalactic bright ( $\mathrm{cm}$ wavelength selected) radio source population $(\sim 10 \%$ and $\sim 20 \%$, respectively), but are not well understood (e.g. O'Dea 1998). They are powerful but compact radio sources whose spectra are generally simple and convex with peaks near $1 \mathrm{GHz}$ and $100 \mathrm{MHz}$ respectively. The GPS sources are entirely contained within the extent of the nuclear narrow line region ( $\$ 1 \mathrm{kpc}, \mathrm{NLR})$ while the CSS sources are contained entirely within the host galaxy $(\$ 15 \mathrm{kpc})$. GPS and CSS sources are important because (1) they probe the NLR and interstellar medium (ISM) of the host galaxy and (2) they may be the younger stages of powerful large-scale radio sources - giving us insight into radio source genesis and evolution.

The currently favored hypothesis is that the GPS and CSS sources are indeed the young progenitors of the large scale powerful double sources (e.g. Carvalho 1985; Hodges \& Mutel 1987; Begelman 1996; Fanti et al. 1995; Readhead et al. 1996; O'Dea 1998; Snellen et al. 2000; Alexander 2000). In this model they propagate relatively quickly through the ISM of the parent galaxy with advance speeds of a few percent of the speed of light. Observed proper motions tend to be a bit higher - in the range $0.05-0.2 h^{-1}$ c, e.g., Polatidis \& Conway (2003), though the detections may be biased towards objects with the highest velocities.

Searches for $21 \mathrm{~cm} \mathrm{H} \mathrm{I} \mathrm{absorption} \mathrm{have} \mathrm{produced} \mathrm{a} 50 \%$ detection rate in GPS and CSS sources (Vermeulen et al. 2003; Pihlström et al. 2003) in contrast to normal elliptical radio 
galaxies, where the detection rate does not exceed $\sim 10 \%$ (van Gorkom et al. 1989). This indicates that clouds of atomic hydrogen are very common in the environments of GPS and CSS sources or that the geometry and/or morphology of GPS and CSS sources is very favorable for the detection of $\mathrm{HI}$ absorption. The close alignment and similar spatial extents of the radio continuum and optical emission line plasma suggests the existence of a close coupling between the thermal gas and the radio sources (de Vries et al. 1997, 1999; Axon et al. 2000). The broad and highly structured spatially integrated [OIII] $\lambda 5007$ line widths observed by Gelderman \& Whittle (1994) strongly suggest that the radio source is dominating the emission line kinematics. This has been confirmed by Hubble Space Telescope spectroscopy (O'Dea et al. 2002).

Thus, the simple picture of evolution may require the incorporation of interaction of the radio sources with dense gas clouds in their ISM (e.g., Jeyakumar et al. 2005). In order to probe the nature of the relationship between the gas clouds and the radio source we have obtained high resolution EVN observations of the redshifted $21 \mathrm{~cm}$ line seen in absorption against 1 GPS and 2 CSS radio galaxies in our WSRT spectra (Vermeulen et al. 2003). The results for $2050+364$ are presented by Vermeulen et al. (2006). Here we present the results for 3C 49 and 3C 268.3.

\section{Observations and data reduction}

The $21 \mathrm{~cm} \mathrm{HI}$ absorption lines associated with the sources 3C $49(z=0.6207,876.7 \mathrm{MHz})$ and 3C $268.3(z=$ $0.37116,1035.1 \mathrm{MHz})^{1}$ were observed on 1999 September 14 and 09 , respectively, for about $7 \mathrm{~h}$ each, using the UHF receivers $(800-1300 \mathrm{MHz})$ on the European VLBI Network (EVN). The sources J0249+063, 3C 84, J1048+717, 3C 286, DA 406, and 3C 454.3 were used as calibrators. The recorded bandwidth of $4 \mathrm{MHz}$ was correlated at the NRAO, Socorro correlator with 256 spectral channels, for a resolution of $5.3 \mathrm{~km} \mathrm{~s}^{-1}$ and $4.5 \mathrm{~km} \mathrm{~s}^{-1}$ in 3C 49 and 3C 268.3, respectively.

At the time of these observations, we could only obtain data in left and right circular polarisations from the Effelsberg and Westerbork (Tied Array) telescopes, and two orthogonal linear polarisations from the Onsala (25 m) telescope. For 3C 268.3, we obtained left circularly polarised data from the Lovell Telescope at Jodrell Bank in addition.

The NRAO Astronomical Image Processing Software (AIPS) package was used for the initial data processing (fringe fitting, spectral passband calibration and a priori gain calibration). The Caltech DIFMAP package (Shepherd 1997) was used for all further calibration and analysis (Cleaning, self-calibration, model-fitting). The data processing and analysis steps used to obtain final continuum images and radio spectra towards the various features in these images are explained in detail in our 2050+364 EVN UHF observations paper (Vermeulen et al. 2006). It should be noted in particular that all available polarization products were averaged together, including the cross-correlations between linear and circular

\footnotetext{
${ }^{1}$ Redshifts from Spinrad et al. (1985) for 3C 49 and Gelderman \& Whittle (1994) for 3C 268.3.
}

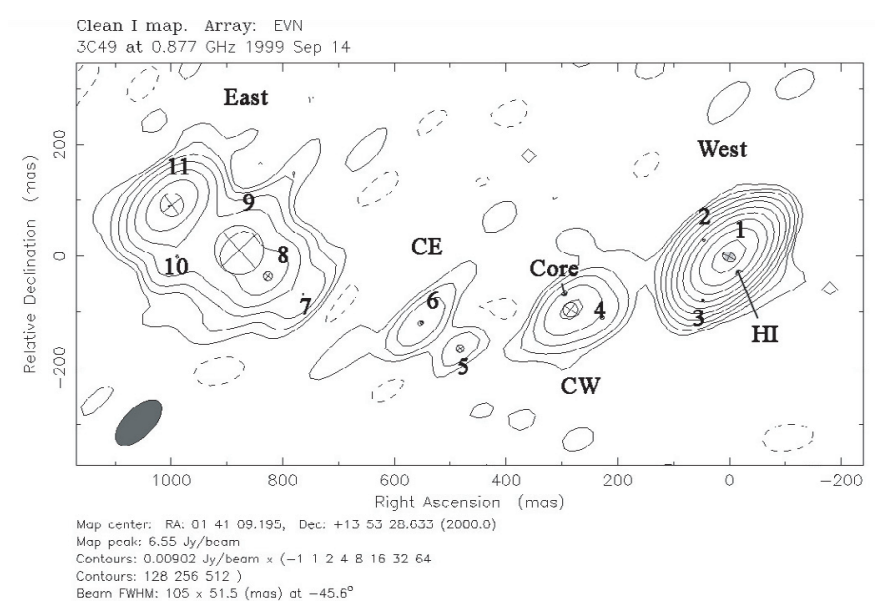

Fig. 1. Clean map of $3 \mathrm{C} 49$ with the numbered Gaussian components drawn on top. We use letters for the major source regions (from left to right: $\mathrm{E}, \mathrm{CE}, \mathrm{CW}, \mathrm{W})$. The core of the source is in the center of the CW component (Ludke et al. 1998).

polarisations. We believe the sensitivity gained to total intensity is more important than resultant limitations on absolute flux calibration accuracy or on (image or spectral) dynamic range; we think these are more affected by the sparseness of the array and the lack of complete system temperature data and gain curves. In fact, we caution that the absolute flux density scales for the data shown are uncertain even at the $50 \%$ level $^{2}$. However, the main astrophysical results are unaffected, since they depend on opacities rather than on absolute flux densities. Furthermore, great effort was expended to obtain reliable relative (self)calibrations between the telescopes, in an extensive series of very gradual self-calibration iterations.

We believe the continuum structures and spectral line profiles obtained are robust against the overall calibration uncertainties. Indeed, the continuum structures obtained at these novel frequencies correspond well with those found at other frequencies, as discussed in Sect. 3.1. In order to restrict the number of free parameters, the sky model fitted to the visibility data during the self-calibration cycles consisted of a limited number of Gaussian components, which are shown overplotted on the continuum images in Figs. 1 and 2; their parameters, fitted to the visibility data, are given in Tables 2 and 3 .

\subsection{Spectrum analysis}

Given the sparseness of these VLBI datasets, the most robust way to derive line spectra of the various regions of the sources was to re-fit, separately for each of the line channels, the flux density of each of the Gaussian model components, as first derived for the line-free continuum. The positions and diameters of the model components were fixed at the continuum values. Resultant spectra are shown in Figs. 3 to 6 for the locations where absorption was detected. Showing percentage absorption depth or opacity with respect to the continuum strength of the appropriate components avoids the uncertainties in the absolute flux density scale discussed above. Zero velocity was

2 This error is not included in our reported errors on flux densities. 


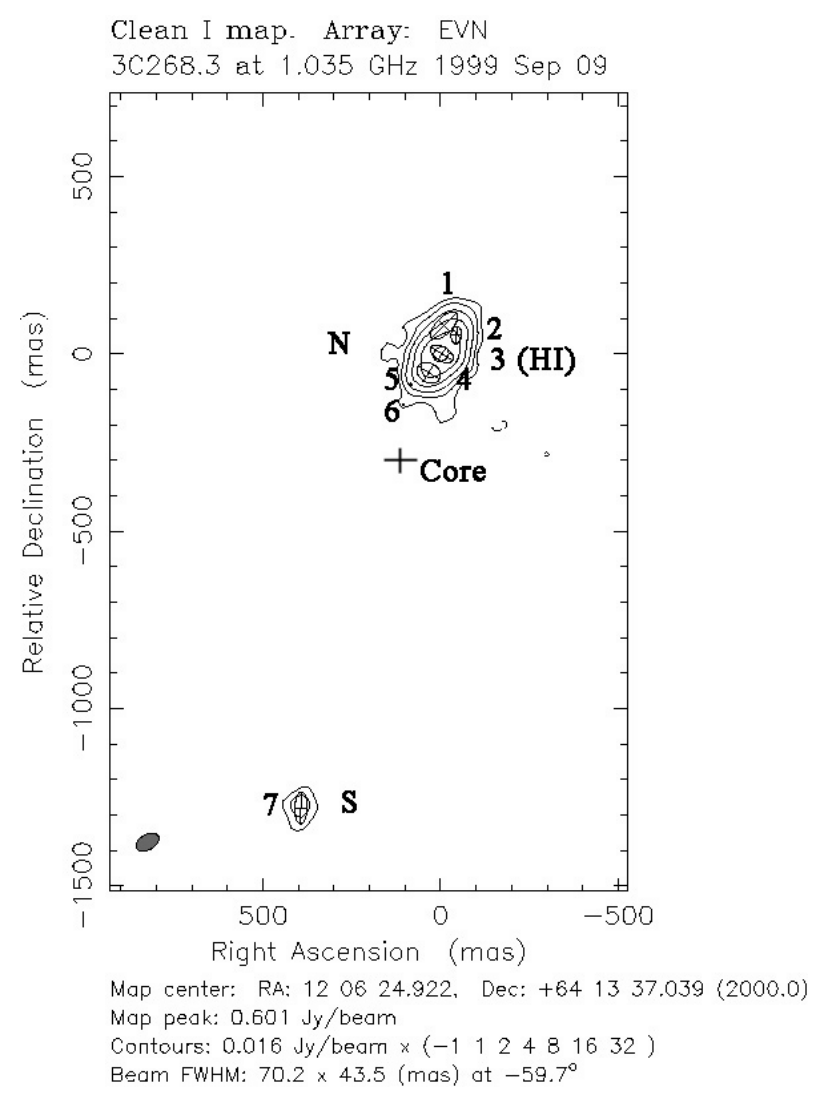

Fig. 2. Clean map of $3 \mathrm{C} 268.3$ with the Gaussian components drawn on top. We use numbers to refer to the Gaussian model components (1 to 7, from top to bottom, being 1 the furthest north, and 7 the furthest south) and letters for the source real components ( $\mathrm{N}$ for the northern, $\mathrm{S}$ for the southern). The position of the core detected at $5 \mathrm{GHz}$ by (Ludke et al. 1998) has been marked with a cross.

taken to be at the nominal optical redshift found in the literature; all calculations used the appropriate relativistic formulae.

We fitted Gaussian profiles to the absorption towards our sources using standard, non-linear $\chi^{2}$ minimization techniques. We measured the optical depth of each line (Figs. 5 and 6), its full width at half maximum ( $F W H M$ ), and its centroid. The values are listed with their formal fitting errors in Tables 2 and 3. The reduced $\chi^{2}$ indicated that two Gaussian lines were needed to fit the absorption towards 3C 49 (Fig. 3), while one was sufficient for 3C 268.3 (Fig. 4). For all locations where the spectra showed no detectable absorption, $2 \sigma$ limits have been estimated from the spectral rms noise level, assuming that any putative line would occur at a similar velocity as the line detected for the same source, and with the same FWHM as for the detection (the average of the two detections for $3 \mathrm{C} 49$ ). The resultant limits are given in Table 3.

\section{Results}

We have detected localized $21 \mathrm{~cm} \mathrm{HI}$ absorption regions in both 3C 49 and 3C 268.3 consistent with the previously published WSRT observations (Vermeulen et al. 2003). The higher resolution of our VLBI observations allows us to locate the $\mathrm{HI}$ absorber and propose a physical scenario. We have studied the continuum milliarcsecond structure of the sources, as well as the properties of the $\mathrm{HI}$ absorption-line gas. Figures 1 and 2 show the clean maps, the Gaussian components used to model them, and the location of the HI absorption. The coordinates $(0,0)$ were driven by self-calibration to coincide with the brightest pixel in the image, rather than with the location of the brightest Gaussian component. Tables 2 and 3 list the details of all the Gaussian source components and their fitted absorption line properties.

\subsection{The milliarcsecond scale continuum structure}

These radio sources have been been previously imaged with high angular resolution (e.g., Ludke et al. 1998; Sanghera et al. 1995; Neff et al. 1995; van Breugel et al. 1992; Nan et al. 1991; Akujor et al. 1991). Our small array of telescopes did not allow us to perform a high quality calibration but we obtained continuum maps and structures consistent with those previously published.

\subsubsection{C 49}

3C 49 is a CSS galaxy (Spinrad et al. 1985) at a redshift $z=$ 0.6207 with radio components extended mostly east-west over a total angular size of 1 arcsec or $\simeq 6.8 \mathrm{kpc}^{3}$.

We detect the three components (labeled East, Center-West (CW) and West) which have been seen previously (i.e. Nan et al. 1991; van Breugel et al. 1992; Neff et al. 1995; Sanghera et al. 1995; Ludke et al. 1998). Multifrequency observations have shown that $\mathrm{CW}$ contains the flat spectrum core (Sanghera et al. 1995; Ludke et al. 1998). We also find an additional component labeled Center-East (CE) whose reality is uncertain. The CE component is faint but its inclusion in the clean components seemed to improve the final image. West is the brightest component in the map (integrated flux density $=7.48 \pm 0.06 \mathrm{Jy}$ ), which is roughly 66,33 and 4.5 times more than the integrated flux density of $\mathrm{CE}, \mathrm{CW}$ and East respectively.

\subsection{2. $3 \mathrm{C} 268.3$}

3C 268.3 is a CSS galaxy (Spinrad et al. 1985; Gelderman \& Whittle 1994) at a redshift $z=0.37116$, with two main radio components oriented roughly north-south with a total angular size of 1.5 arcsec or $\simeq 7.6 \mathrm{kpc}$ (i.e., Nan et al. 1991; van Breugel et al. 1992; Neff et al. 1995; Sanghera et al. 1995; Ludke et al. 1998). Our observations are consistent with the previous results. Ludke et al. (1998) have found a faint, possible core at $5 \mathrm{GHz}$, about $1.5 \mathrm{kpc}$ south of the northern lobe.

The northern component has an integrated flux density of $2.25 \pm 0.01 \mathrm{Jy}$, which is roughly 22 times more than the southern component. The northern component also shows a more complex structure: 6 Gaussian components were required for it, as opposed to a single one for the southern feature.

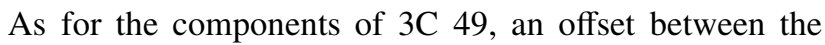
brightest Gaussian component and the image peak brightness of 3C 268.3 is observed. In this case, the offset is even

\footnotetext{
${ }^{3} \mathrm{Ho}=71 \mathrm{~km} \mathrm{~s}^{-1} \mathrm{Mpc}^{-1}, \Omega_{\mathrm{M}}=0.27$ and $\Omega_{\Lambda}=0.73$.
} 
Table 1. Observational parameters of the sources.

\begin{tabular}{|c|c|c|c|c|c|c|c|}
\hline \multirow[b]{2}{*}{ Source } & \multirow{2}{*}{$\begin{array}{l}\text { Freq. } \\
(\mathrm{GHz})\end{array}$} & \multirow{2}{*}{$\begin{array}{l}\text { Time on source } \\
\text { hh:mm:ss }\end{array}$} & \multirow{2}{*}{$\begin{array}{l}\text { Number } \\
\text { of scans }\end{array}$} & \multirow{2}{*}{$\begin{array}{c}\mathrm{rms} \\
\left(\mathrm{Jy} \mathrm{beam}^{-1}\right)\end{array}$} & \multirow{2}{*}{$\begin{array}{c}\text { Cont. Peak } \\
\left(\text { Jy beam }^{-1}\right)\end{array}$} & \multicolumn{2}{|c|}{ Restoring beam } \\
\hline & & & & & & (mas) & $(\mathrm{deg})$ \\
\hline $3 \mathrm{C} 49$ & 0.8767 & $07: 26: 18$ & 4 & $2.5 \times 10^{-3}$ & 6.550 & $105 \times 51.5$ & -45.6 \\
\hline $3 C 268.3$ & 1.0351 & 06:59:14 & 4 & $1.4 \times 10^{-3}$ & 0.601 & $70.2 \times 43.5$ & 59.7 \\
\hline
\end{tabular}

Summary of the EVN observations of 3C 49 and 3C 268.3, in September, 1999. The first column gives the name of the source, the second column the frequency of our observations, the third column the exposure time, the fourth the number of scans made on each particular source (the exposure time shown is the sum of all the scans). The RMS column is the noise in an empty region of the map. Cont. Peak is the peak flux density of the continuum image. The Beam column gives the FWHM and orientation of the beam.

Table 2. Components and absorption line properties. Detections.

\begin{tabular}{ccccccccccccc}
\hline \hline Source & $\mathrm{GC}$ & $\begin{array}{c}R \\
(\mathrm{mas})\end{array}$ & $\begin{array}{c}\theta \\
(\mathrm{deg})\end{array}$ & $\begin{array}{c}\text { Major Minor } \\
(\mathrm{mas})\end{array}$ & $\begin{array}{c}\text { Flux }^{a} \\
(\mathrm{mas})\end{array}$ & $\mathrm{SC}$ & $\Delta S / S_{\text {cont }}$ & $\begin{array}{c}F W H M \\
(\mathrm{Jy})\end{array}$ & & $\tau^{b}$ & $\begin{array}{c}N_{\mathrm{HI}}{ }^{b} \\
\left(\mathrm{~km} \mathrm{~s}^{-1}\right)\end{array}$ & $\begin{array}{c}\text { Center } \\
\left(0^{20}\left(T_{\mathrm{S}} / 100 \mathrm{~K}^{\mathrm{K}} \mathrm{cm}^{-2}\right.\right.\end{array}$ \\
\hline $3 \mathrm{C} 49$ & $1(\mathrm{~W})$ & 1.24 & 133 & 24 & 24 & $7.28 \pm 0.03$ & 1 & $3.5 \% \pm 0.4 \%$ & $20.9 \pm 3.7$ & $0.036 \pm 0.003$ & $1.5 \pm 0.3$ & $-138 \pm 2 \pm 19$ \\
$3 \mathrm{C} 49$ & $1(\mathrm{~W})$ & - & - & - & - & - & 2 & $1.9 \% \pm 0.4 \%$ & $22.7 \pm 8.1$ & $0.019 \pm 0.006$ & $0.8 \pm 0.4$ & $-160 \pm 5 \pm 19$ \\
$3 \mathrm{C} 268.3$ & $3(\mathrm{~N})$ & 3.9 & -88 & 72 & 39 & $1.10 \pm 0.01$ & - & $2.5 \% \pm 0.6 \%$ & $67.1 \pm 6.0$ & $0.025 \pm 0.005$ & $3.2 \pm 0.6$ & $+190 \pm 2 \pm 12$ \\
\hline
\end{tabular}

Columns 2 to 7 list the properties of the fitted Gaussian components $(G C)$ : distance (to coordinates $(0,0)$ ) and position $(R, \theta)$, axes' size $($ Major, Minor), and integrated flux density. Columns 8 to 13 list the properties of the H I absorptions: spectral component (SC): peak depth divided by continuum level $\left(\triangle S / S_{\text {cont }}\right)$, width $(F W H M)$, optical depth $(\tau)$, column density $\left(N_{\mathrm{H}_{\mathrm{I}}}\right)$ and central wavelength $($ Center $)$.

${ }^{a}$ The errors in the absolute flux density scales include only the formal statistical error, and do not include the errors due to sparseness of the array (up to $50 \%$, see Sect. 2).

${ }^{b}$ Not taking into account the covering factor (see Sect. 3.2).

Table 3. Components and absorption line properties. Non detections.

\begin{tabular}{|c|c|c|c|c|c|c|c|c|c|c|}
\hline Source & $\mathrm{MC}$ & $\begin{array}{c}R \\
\text { (mas) }\end{array}$ & $\begin{array}{c}\theta \\
(\mathrm{deg})\end{array}$ & $\begin{array}{l}\text { Major } \\
\text { (mas) }\end{array}$ & $\begin{array}{l}\text { Minor } \\
\text { (mas) }\end{array}$ & $\begin{array}{l}\text { Flux }^{a} \\
(\mathrm{Jy})\end{array}$ & $\Delta S / S_{\text {cont }}$ & $\begin{array}{l}F W H M \\
\left(\mathrm{~km} \mathrm{~s}^{-1}\right)\end{array}$ & $\tau^{b}$ & $\begin{array}{c}N_{\mathrm{HI}}{ }^{b} \\
10^{20}\left(T_{\mathrm{S}} / 100 \mathrm{~K}\right) \mathrm{cm}^{-2}\end{array}$ \\
\hline $3 \mathrm{C} 49$ & $2(W)$ & 54.5 & 58 & 4 & 4 & $0.13 \pm 0.02$ & $<31 \%$ & 21.8 & $<0.37$ & $<16$ \\
\hline $3 \mathrm{C} 49$ & $3(\mathrm{~W})$ & 92.6 & 149 & 4 & 4 & $0.05 \pm 0.02$ & $<100 \%$ & 21.8 & $X$ & $X$ \\
\hline $3 \mathrm{C} 49$ & $4(\mathrm{CW})$ & 300 & 108 & 40 & 40 & $0.23 \pm 0.02$ & $<19 \%$ & 21.8 & $<0.21$ & $<8.7$ \\
\hline $3 \mathrm{C} 49$ & $5(\mathrm{CE})$ & 509 & 109 & 14 & 14 & $0.04 \pm 0.02$ & $<100 \%$ & 21.8 & $X$ & $\mathrm{X}$ \\
\hline $3 \mathrm{C} 49$ & $6(\mathrm{CE})$ & 564 & 102 & 6 & 6 & $0.07 \pm 0.02$ & $<57 \%$ & 21.8 & $<0.85$ & $<36$ \\
\hline $3 C 49$ & $7(\mathrm{E})$ & 767 & 95 & 3 & 3 & $0.07 \pm 0.02$ & $<57 \%$ & 21.8 & $<0.85$ & $<36$ \\
\hline $3 \mathrm{C} 49$ & $8(\mathrm{E})$ & 827 & 93 & 16 & 16 & $0.21 \pm 0.03$ & $<30 \%$ & 21.8 & $<0.36$ & $<15$ \\
\hline $3 C 49$ & $9(\mathrm{E})$ & 878 & 89 & 88 & 88 & $0.46 \pm 0.04$ & $<17 \%$ & 21.8 & $<0.19$ & $<8.1$ \\
\hline $3 \mathrm{C} 49$ & $10(\mathrm{E})$ & 989 & 90 & 5 & 5 & $0.11 \pm 0.02$ & $<36 \%$ & 21.8 & $<0.45$ & $<19$ \\
\hline $3 C 49$ & $11(\mathrm{E})$ & 1003 & 85 & 41 & 41 & $0.81 \pm 0.03$ & $<7.4 \%$ & 21.8 & $<0.08$ & $<3.3$ \\
\hline $3 \mathrm{C} 49$ & East Int. & - & - & - & - & $1.66 \pm 0.04$ & $<5.0 \%$ & 21.8 & $<0.05$ & $<2.1$ \\
\hline $3 C 49$ & CE Int. & - & - & - & - & $0.11 \pm 0.04$ & $<63 \%$ & 21.8 & $<1.00$ & $<42$ \\
\hline $3 C 268.3$ & $1(\mathrm{~N})$ & 80 & -7.2 & 100 & 45 & $0.24 \pm 0.01$ & $<10 \%$ & 67.1 & $<0.11$ & $<14$ \\
\hline $3 C 268.3$ & $2(\mathrm{~N})$ & 69 & -40 & 49 & 30 & $0.31 \pm 0.01$ & $<6.0 \%$ & 67.1 & $<0.06$ & $<8.1$ \\
\hline $3 C 268.3$ & $4(\mathrm{~N})$ & 62 & 148 & 71 & 55 & $0.57 \pm 0.02$ & $<3.0 \%$ & 67.1 & $<0.03$ & $<4.0$ \\
\hline $3 C 268.3$ & $5(\mathrm{~N})$ & 177 & 143 & 3 & 3 & $0.020 \pm 0.007$ & $<56 \%$ & 67.1 & $<0.82$ & $<107$ \\
\hline $3 C 268.3$ & $6(\mathrm{~N})$ & 120 & 135 & 6 & 6 & $0.01 \pm 0.01$ & $<100 \%$ & 67.1 & X & $\mathrm{X}$ \\
\hline $3 C 268.3$ & $7(S)$ & 1339 & 162 & 90 & 35 & $0.104 \pm 0.007$ & $<13 \%$ & 67.1 & $<0.14$ & $<18$ \\
\hline
\end{tabular}

As for Table 2 but for non detections. The numbers shown correspond to the $2 \sigma$ detection limits (maximum peak depth $=2 \times$ rms/continuum). An "X" represents no value, as the absorption could be $100 \%$. Rows 11 and 12, East Int, CE Int, correspond to the spectrum spatially integrated over the entire Eastern and CE component of 3C 49 respectively. For 3C 268.3, the South component consists of a single Gaussian component (7).

${ }^{a}$ The errors in the absolute flux density scales include only the formal statistical error, but do not include the errors due to sparseness of the array (up to $50 \%$, see Sect. 2).

${ }^{b}$ Not taking into account the covering factor (see Sect. 3.2). 


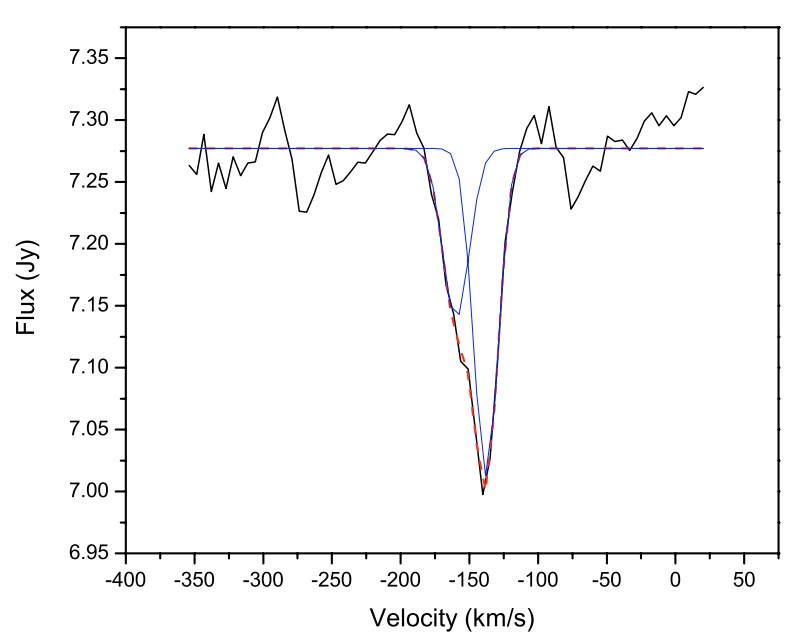

Fig. 3. Spectrum of the absorption in the West component of $3 \mathrm{C} 49$, with the de-blended lines and total fit over plotted.

bigger, which is consistent with the North component being reproduced by more, brighter and bigger Gaussian components than $\mathrm{W}$ in $3 \mathrm{C} 49$.

\subsection{The HI absorption}

We have detected $21 \mathrm{~cm} \mathrm{H}$ I absorption against the western lobe of $3 \mathrm{C} 49$ and the northern lobe of $3 \mathrm{C} 268.3$. Figures 3, 4, 5 and 6 show the spectra and optical depth of these regions in 3C 49 and $3 \mathrm{C} 268.3$.

The measured peak depth $\Delta S$ of the absorption line depends on both the optical depth $\tau$ and the covering factor of the hydrogen $c_{f}$ (e.g. Wolfe \& Burbidge 1975),

$c_{f}=\frac{\Delta S}{S\left(1-\mathrm{e}^{-\tau}\right)} \quad$ or $\quad \tau=-\ln \left(1-\frac{\Delta S}{S c_{f}}\right)$

where $S$ is the continuum flux density. For a uniform source there is a minimum covering factor required by the observed ratio of line depth to continuum flux density $c_{f}>\Delta S / S$ (e.g. O'Dea et al. 1994), and for a complete covering of the source $c_{f}=1$.

The column density, $N_{\mathrm{HI}}$, is given by:

$N_{\mathrm{HI}}=0.18 \times 10^{21}\left(T_{\mathrm{S}} / 100 \mathrm{~K}\right) \int \tau_{v} \mathrm{~d} v \mathrm{~cm}^{-2}$

(e.g., Dopita \& Sutherland 2003; O'Dea et al. 1994), where $T_{\mathrm{s}}$ is the spin temperature, $\tau$ the optical depth, and $v$ is the velocity. For a Gaussian profile:

$N_{\mathrm{HI}} \simeq 1.94 \times 10^{20}\left(T_{\mathrm{S}} / 100 \mathrm{~K}\right) \tau_{0} \Delta V \mathrm{~cm}^{-2}$

where $\tau_{0}$ is the peak optical depth in the line and $\Delta V$ is the $F W H M$ of the Gaussian line profile. A spin temperature $T_{\mathrm{s}}=$ $100 \mathrm{~K}$ is applicable under typical ISM circumstances, although close to an AGN it could be higher (e.g. Morganti et al. 2001).

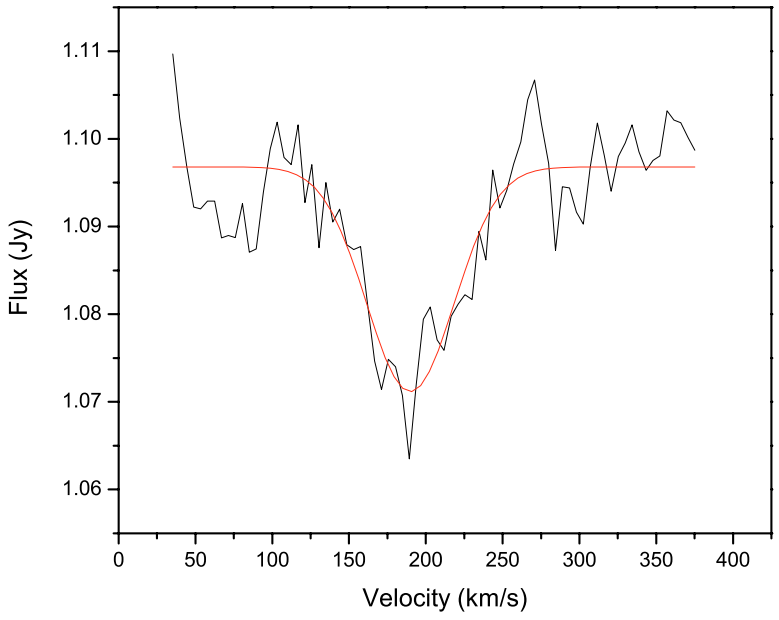

Fig. 4. Spectrum of the absorption in the North component of 3C 268.3, with the fit over-plotted.

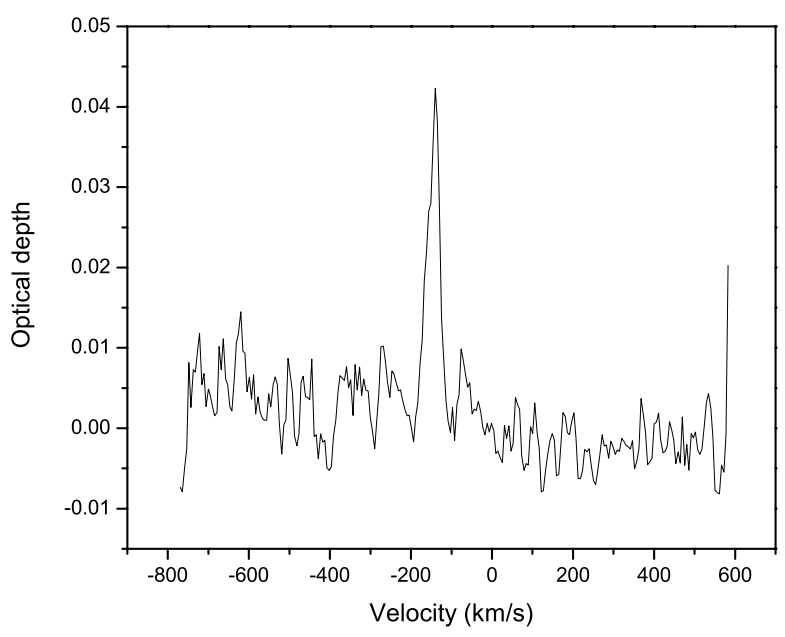

Fig. 5. Optical depth for the for the H I detection in the West component of $3 \mathrm{C} 49$.

\subsubsection{C 49}

3C 49 shows H I absorption in the center of the Western component with a peak depth of $\sim 4 \%$ of the continuum level. The reduced $\chi^{2}$ indicated that two Gaussian lines were needed to fit the absorption with peak depths of $\sim 1.5 \%$ and $\sim 2.1 \%$. The column densities are 1.5 and $0.8 \times 10^{20}\left(T_{\mathrm{S}} / 100 \mathrm{~K}\right) \mathrm{cm}^{-2}$. The absorption seems to be blueshifted in the host galaxy rest frame.

The covering factor, $\mathrm{c}_{f}$ for the absorption towards $\mathrm{W}$ in $3 \mathrm{C} 49$ is $1>c_{f}>0.04$. If we assume the two absorption lines to be produced by spherical clouds of similar radius, equal to the measured $F W H M$ of the component $(24$ mas $=163 \mathrm{pc}$ at the source distance), then the density of these clouds would be $220 / c_{f}\left(T_{\mathrm{s}} / 100\right) \mathrm{cm}^{-3}$ and $160 / c_{f}\left(T_{\mathrm{s}} / 100\right) \mathrm{cm}^{-3}$. 


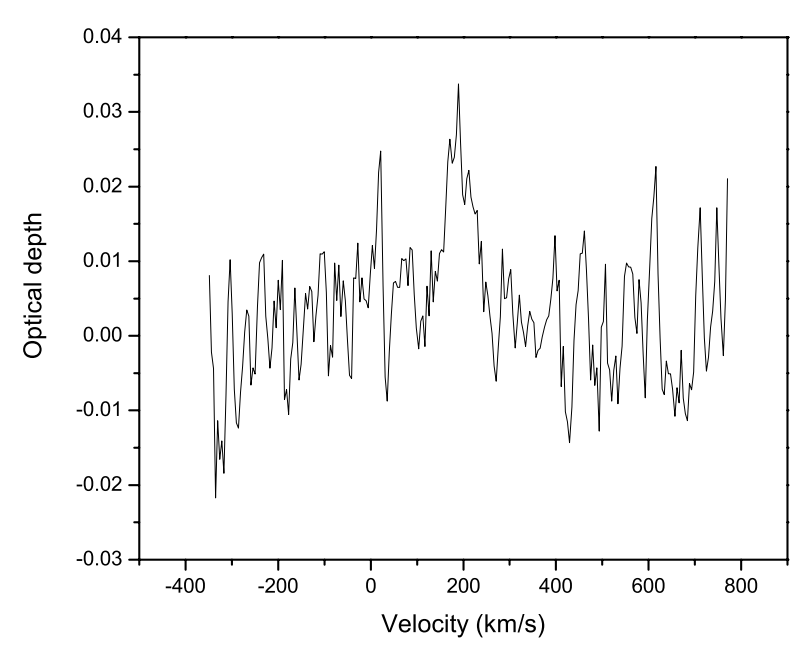

Fig. 6. Optical depth for the H I detection in the North component of $3 \mathrm{C} 268.3$.

\subsubsection{C 268.3}

3C 268.3 shows an absorption profile in the center of the northern lobe. $\chi^{2}$ fitting shows that an adequate representation for the detection is just one Gaussian line redshifted in the host galaxy rest frame; the column depth is $3.2 \times 10^{20}\left(T_{\mathrm{S}} / 100 \mathrm{~K}\right) \mathrm{cm}^{-2}$. Inspection of Table 3 shows that the limits on the other components are too high to rule out the presence of $\mathrm{HI}$ at similar levels in the southern component, which is much fainter than the northern component. The most intriguing $2 \sigma$ detection limit is $3.0 \%$ in component $4(\mathrm{~N})$, which, compared to the detection in component $3(\mathrm{~N})$, suggests that, possibly, the extent of the absorption towards the northern lobe is limited to $72 \times 39$ mas $(367 \times 199 \mathrm{pc}$ at the source distance).

The covering factor, $c_{f}$, for $3 \mathrm{C} 2683$ is $1>c_{f}>0.025$. If we assume the absorption line to be produced by a spherical cloud of a radius equal to the average of the measured $F W H M$ of the component $(55.5 \mathrm{mas}=283 \mathrm{pc})$, then the density of the cloud would be $360 / c_{f}\left(T_{\mathrm{S}} / 100\right) \mathrm{cm}^{-3}$.

\section{Discussion}

We have detected $\mathrm{HI}$ absorption towards the western radio lobe of 3C 49 and the northern lobe of $3 \mathrm{C} 268.3$. There are several possible hypotheses for the nature of the absorbing gas; e.g., (1) unrelated foreground clouds (e.g., in the ISM of the host galaxy) (2) an organized structure in the host galaxy such as a disk (as discussed by Pihlström et al. 2003), and (3) clouds which are interacting with the radio jet. We argue here that our results are consistent with the $\mathrm{H}$ I being produced in clouds which are in the environment of the radio source.

In $3 \mathrm{C} 49$, the west lobe is $\sim 4.5$ times brighter than the East lobe and $\sim 3$ times closer to the core. Both radio lobes are unpolarized (Ludke et al. 1998). The emission line gas imaged by HST is very faint but seems to be asymmetric and is brighter near the west lobe (de Vries et al. 1997, 1999; Axon et al. 2000).
In $3 \mathrm{C} 268.3$, the northern radio lobe is roughly 22 times brighter than the southern lobe and $\sim 3$ times closer to the core. In addition, Ludke et al. (1998) show that the radio polarization is asymmetric and that the northern lobe is much more strongly depolarized than the southern lobe. The emission line gas imaged by HST is also asymmetrically distributed and is much brighter near the northern lobe, while the southern lobe seems to extend beyond the line emission (de Vries et al. 1997, 1999; Axon et al. 2000).

These correlated asymmetries in the radio and optical line emission are similar to those seen by McCarthy et al. (1991) in samples of powerful high redshift radio galaxies. We suggest that these data are consistent with a picture in which one side of the radio source is strongly interacting with dense clouds of gas. The interaction causes the lobe to propagate more slowly, resulting in a smaller separation distance from the core (e.g., De Young 1991; Carvalho 1998; Jeyakumar et al. 2005). The higher radio luminosity could be due to smaller adiabatic expansion losses or to increased energy production efficiency due to compression and shocks (e.g., Jeyakumar et al. 2005). The clouds will also act as a Faraday screen, causing the observed depolarization as seen in 3C 268.3. The association of the optical emission line clouds and the $\mathrm{HI}$ absorption suggests that the $\mathrm{HI}$ absorption is produced in the atomic cores of the clouds which are seen in the emission line images (as may be the case in PKS2322-123, O'Dea et al. 1994). However, we caution that the data do not exclude the existence of H I on both sides of 3C 49 and 3C 268.3. An alternate scenario would be that the brighter lobe is beamed towards us and we are seeing H I absorption produced in clouds which are swept up by the radio lobes. However, if the brightness asymmetry is due to beaming we would expect the brighter lobe to be further from the core than the weaker lobe (due to the difference in light travel time), which is the opposite of what is observed.

A study of the emission line nebulae in three CSS sources - 3C 67, 3C 277.1 and 3C 303.1 - using HST long slit spectroscopy has shown that the kinematics of the gas are consistent with the clouds having been accelerated to velocities of several hundred $\mathrm{km} / \mathrm{s}$ by shocks induced by the expanding radio lobes (O'Dea et al. 2002). The ionization diagnostics of the gas in CSS sources are also consistent with a contribution from shock-ionized gas (Labiano et al. 2005; Morganti et al. 1997; Gelderman \& Whittle 1994). Thus, the observed blue and redshifts of the H I clouds of several hundred $\mathrm{km} / \mathrm{s}$ in $3 \mathrm{C} 49$ and 3C 268.3 could be attributed to bow shock induced velocities. It seems likely that both positive and negative velocities can result, depending on whether the HI absorption occurs in gas pushed towards the observer or in gas being entrained away from the observer.

\section{Summary}

We present European VLBI Network spectral line observations in the UHF band of the redshifted $21 \mathrm{~cm} \mathrm{H}$ I line in two compact steep spectrum radio galaxies. We have detected H I absorption towards the western radio lobe of $3 \mathrm{C} 49$ and the northern lobe of 3C 268.3. The radio lobes with H I absorption (1) are brighter and closer to the core than the opposite lobes; (2) are 
more depolarized (in 3C 268.3); and (3) are preferentially associated with optical emission line gas. The association between the $\mathrm{H}$ I absorption and the emission line gas, supports the hypothesis that the $\mathrm{H}$ a absorption is produced in the atomic cores of the emission line clouds, but we cannot rule out the existence of H I elsewhere. We suggest that the asymmetries in the radio and optical emission are due to interaction of the radio source with an asymmetric distribution of dense clouds in their environment. Our results are consistent with a picture in which CSS sources interact with clouds of dense gas as they propagate through their host galaxy.

Acknowledgements. This research has made use of the NASA/IPAC Extragalactic Database (NED) which is operated by the Jet Propulsion Laboratory, California Institute of Technology, under contract with the National Aeronautics and Space Administration. This research has made use of NASA's Astrophysics Data System. The European VLBI Network is a joint facility of European, Chinese, South African and other radio astronomy institutes funded by their national research councils.

\section{References}

Akujor, C. E., Spencer, R. E., Zhang, F. J., et al. 1991, MNRAS, 250, 215

Alexander, P. 2000, MNRAS, 319, 8

Axon, D. J., Capetti, A., Fanti, R., et al. 2000, AJ, 120, 2284

Begelman, M. C. 1996, in Cygnus A - Study of a Radio Galaxy, 209

Carvalho, J. C. 1985, MNRAS, 215, 463

Carvalho, J. C. 1998, A\&A, 329, 845

de Vries, W. H., O'Dea, C. P., Baum, S. A., et al. 1997, ApJS, 110, 191

de Vries, W. H., O’Dea, C. P., Baum, S. A., \& Barthel, P. D. 1999, ApJ, 526, 27

De Young, D. S. 1991, ApJ, 371, 69

Dopita, M. A., \& Sutherland, R. S. 2003, Astrophysics of the diffuse universe, Astrophysics of the diffuse universe (Berlin, New York: Springer), Astronomy and astrophysics library

Fanti, C., Fanti, R., Dallacasa, D., et al. 1995, A\&A, 302, 317

Gelderman, R., \& Whittle, M. 1994, ApJS, 91, 491
Hodges, M. W., \& Mutel, R. L. 1987, in Superluminal Radio Sources, $168-173$

Jeyakumar, S., Wiita, P. J., Saikia, D. J., \& Hooda, J. S. 2005, A\&A, 432,823

Labiano, A., O’Dea, C. P., Gelderman, R., et al. 2005, A\&A, 436, 493

Ludke, E., Garrington, S. T., Spencer, R. E., et al. 1998, MNRAS, 299, 467

McCarthy, P. J., van Breugel, W., \& Kapahi, V. K. 1991, ApJ, 371, 478

Morganti, R., Tadhunter, C. N., Dickson, R., \& Shaw, M. 1997, A\&A, 326,130

Morganti, R., Oosterloo, T. A., Tadhunter, C. N., et al. 2001, MNRAS, 323,331

Nan, R., Schilizzi, R. T., Fanti, C., \& Fanti, R. 1991, A\&A, 252, 513

Neff, S. G., Roberts, L., \& Hutchings, J. B. 1995, ApJS, 99, 349

O'Dea, C. P. 1998, PASP, 110, 493

O'Dea, C. P., Baum, S. A., \& Gallimore, J. F. 1994, ApJ, 436, 669

O'Dea, C. P., de Vries, W. H., Koekemoer, A. M., et al. 2002, AJ, 123, 2333

Pihlström, Y. M., Conway, J. E., \& Vermeulen, R. C. 2003, A\&A, 404, 871

Polatidis, A. G., \& Conway, J. E. 2003, Publications of the Astronomical Society of Australia, 20, 69

Readhead, A. C. S., Taylor, G. B., Pearson, T. J., \& Wilkinson, P. N. 1996, ApJ, 460, 634

Sanghera, H. S., Saikia, D. J., Luedke, E., et al. 1995, A\&A, 295, 629

Shepherd, M. C. 1997, in Astronomical Data Analysis Software and Systems VI, ASP Conf. Ser., 125, 77

Snellen, I. A. G., Schilizzi, R. T., Miley, G. K., et al. 2000, MNRAS, 319,445

Spinrad, H., Marr, J., Aguilar, L., \& Djorgovski, S. 1985, PASP, 97, 932

van Breugel, W. J. M., Fanti, C., Fanti, R., et al. 1992, A\&A, 256, 56

van Gorkom, J. H., Knapp, G. R., Ekers, R. D., et al. 1989, AJ, 97, 708

Vermeulen, R. C., Pihlström, Y. M., Tschager, W., et al. 2003, A\&A, 404, 861

Vermeulen, R. C., Labiano, A., Barthel, P. D., et al. 2006, A\&A, 447, 489

Wolfe, A. M., \& Burbidge, G. R. 1975, ApJ, 200, 548 